\title{
Daptomycin-induced eosinophilic pneumonia - a systematic review
}

Priyasha Uppal ${ }^{1}$, Kerry L. LaPlante ${ }^{1,2,3}$, Melissa M. Gaitanis ${ }^{1,3}$, Matthew D. Jankowich ${ }^{1,3}$ and Kristina E. Ward ${ }^{2 *}$

\begin{abstract}
Purpose: Eosinophilic pneumonia comprises a group of lung diseases in which eosinophils appear in increased numbers in the lungs and sometimes in the bloodstream. Several case reports link daptomycin use to this phenomenon.

Summary: We performed a systematic literature review to identify cases of eosinophilic pneumonia associated with daptomycin use. Relevant studies were identified by searching Pubmed/Medline, EMBASE, Google Scholar, Cochrane Database of Systematic Reviews, and Clin-Alert from inception to May 2016, and manual searches of reference lists. All case reports that include information regarding patient age, indication, clinical and objective findings, treatment and outcome were evaluated. Abstracts from conference proceedings as well as case reports not in English were excluded. Descriptive statistics were used to analyze the data. Thirty-five patient-cases were included in the final analysis. Patients most likely to be identified with daptomycin-induced eosinophilic pneumonia were male (83\%) and elderly (mean age $65.4 \pm 15$ years). The dose for daptomycin ranged from 4 to $10 \mathrm{mg} / \mathrm{kg} /$ day, but included a large number of patients with renal dysfunction. The average duration of daptomycin therapy upon onset of EP symptoms was $2.8 \pm 1.6$ weeks. Majority of patients presented with dyspnea (94\%), fever (57\%) and were also found to have peripheral eosinophilia (77\%) and infiltrates/opacities of CT/CXR (86\%). Symptom improvement was seen after daptomycin discontinuation (24 h to 1 week). The majority of patients were also prescribed treatment with corticosteroids (66\%).
\end{abstract}

Conclusion: Clinicians should be aware of daptomycin-induced eosinophilic pneumonia and its symptoms along with its presentation and treatment.

Keywords: Daptomycin, Eosinophilia, Pneumonia

\section{Background}

Eosinophilic pneumonia is a rare, but serious respiratory syndrome that occurs when eosinophils accumulate in the lungs $[1,2]$. It has been associated with several medications and chemicals, with antibiotics and nonsteroidal anti-inflammatory drugs among the most common $[3,4]$.

The pathophysiology of acute eosinophilic pneumonia is thought to be caused by detection of an antigen by alveolar macrophages which leads to recruitment of T-helper 2 lymphocytes and subsequent release of interleukin 5 . Interleukin 5 promotes eosinophil production and migration to the lung. Additionally, eotaxin (a potent eosinophil chemoattractant) production by alveolar macrophages, pulmonary endothelial cells, airway smooth muscle cells,

\footnotetext{
* Correspondence: kward@uri.edu

${ }^{2}$ Department of Pharmacy Practice, University of Rhode Island, 7 Greenhouse Rd, Suite 295 J, Kingston, RI 02881, USA

Full list of author information is available at the end of the article
}

and alveolar epithelial cells leads to further accumulation of eosinophils in the lungs [5].

Daptomycin is a cyclic lipopeptide antibiotic derived from the fermentation of Streptomyces roseosporus. Daptomycin has activity against Gram-positive organisms including methicillin-resistant Staphylococcus aureus (MRSA) and vancomycin-resistant enterococci (VRE) [6]. In 2007, pulmonary eosinophilia was added to the "Adverse Reactions, Post-Marketing Experience" section of the product label for daptomycin [2]. A review of the literature and the US FDA Adverse Event Reporting System database in 2012 revealed 7 definite, 13 probable, and 38 possible cases of daptomycin-induced eosinophilic pneumonia [1]. While the mechanism of daptomycin's pulmonary toxicity is not known, the drug undergoes conformational change through interaction with calcium which allows binding to the cytoplasmic membrane, increased membrane permeability, and intracellular ion escape [7]. The antibacterial activity of 
daptomycin is decreased because of its binding to pulmonary surfactant. Some have speculated two potential mechanisms for daptomycin's pulmonary toxicity: 1) chronic daptomycin administration results in drug accumulation near the epithelial alveolar surface causing epithelial injury and pneumonia and 2) the daptomycin-surfactant interaction could alter lipid integrity which may stimulate an inflammatory response $[3,7,8]$.

Per the FDA guidance, eosinophilic pneumonia is attributed to daptomycin when the following criteria are met: 1) concurrent exposure to daptomycin, 2) fever, 3) dyspnea with increased oxygen requirement or requiring mechanical ventilation, 4) new infiltrates on chest $\mathrm{x}$-ray or computed tomography (CT) scan, 5) bronchoalveolar lavage (BAL) with $>25 \%$ eosinophils, and 6 ) clinical improvement following daptomycin withdrawal [2]. Solomon and Schwartz [4] have also developed criteria for drug- or toxin-induced eosinophilic pneumonia that is similar and includes 1) presence of simple, acute, or chronic eosinophilic pneumonia by diagnostic criteria which includes excess of eosinophils either on lung biopsy or BAL (usually $\geq 25 \%$ ) in the setting of parenchymal infiltrates 2) presence of a potential candidate drug or toxin in an appropriate time frame 3) no other cause of eosinophilic pneumonia such as fungal or parasitic infection 4) clinical improvement after cessation of the drug or toxin, and 5) recurrence of eosinophilic pneumonia with re-challenge to the drug or toxin. However, re-challenge is often not recommended as it can be dangerous $[4,5]$.

Although the mechanism of daptomycin-induced eosinophilic pneumonia is unknown, some have speculated that daptomycin may bind to human surfactant and accumulate in the alveolar space causing injury to the epithelium with resulting inflammation $[7,8]$. The purpose of this review is to systematically evaluate the published literature describing daptomycin-induced eosinophilic pneumonia.

\section{Methods}

All relevant cases and studies were identified by systematically searching of the PubMed, EMBASE, Google Scholar, Cochrane Database of Systematic Reviews, and Clin-Alert databases by two reviewers from inception through May 2016. The truncated terms "daptomycin", "eosinophil", and "pneumon" were searched in each database. All case reports that included information regarding patient age, indication, clinical and objective findings, treatment, and outcome were evaluated. Reports not published in English were excluded as well as abstracts from conference proceedings. Descriptive analysis was used to present pooled demographic information and other data where applicable.

\section{Results}

No clinical or observational trials assess daptomycininduced eosinophilic pneumonia; only case reports and case series are published. In 2012, Kim et al. identified 7 definite, 13 probable, and 38 possible cases of daptomycin-induced eosinophilic pneumonia via review of literature and FDA Adverse Event Reporting System Reports (AERS) as defined in Table 1 [1]. Details regarding the 38 possible cases reported through AERS were not described. Of the 20 cases that were identified as definite or probable, 9 have been published in the literature, the remaining 11 were summarized by $\operatorname{Kim}[1,3,9-13]$. We also identified 39 additional cases of eosinophilic pneumonia attributed to daptomycin for a total of 59 cases described in the literature [7, 14-24]. Of those, 21 were excluded from this systematic review because they were abstract presentations at international meetings and did not go through the peer review publishing process [25-41]. Another three were excluded because they were not published in English [42-44]. Currently available data on a total of 35 cases of daptomycin-induced eosinophilic pneumonia is summarized in Table 2.

Table 1 Criteria for inclusion as definite, probably, possible, and unlikely cases of daptomycin-induced eosinophilic pneumonia [1, 2]

\begin{tabular}{|c|c|c|c|}
\hline Definite & Probable & Possible & Unlikely \\
\hline Concurrent exposure to daptomycin & Concurrent exposure to daptomycin & Concurrent exposure to daptomycin & $\begin{array}{l}\text { All other cases that } \\
\text { did not meet criteria }\end{array}$ \\
\hline $\begin{array}{l}\text { Dyspnea with increased oxygen } \\
\text { requirement or requiring mechanical } \\
\text { ventilation }\end{array}$ & $\begin{array}{l}\text { Dyspnea with increased oxygen } \\
\text { requirement or requiring mechanical } \\
\text { ventilation }\end{array}$ & New infiltrates on CXR or CT & \\
\hline New infiltrates on $\mathrm{CXR}$ or $\mathrm{CT}$ & New infiltrates on chest $\mathrm{x}$-ray or $\mathrm{CT}$ & $\begin{array}{l}\text { Clinical improvement following } \\
\text { daptomycin withdrawal OR the patient } \\
\text { died }\end{array}$ & \\
\hline BAL with $>25 \%$ eosinophils & $\begin{array}{l}\text { BAL with } \leq 25 \% \text { eosinophils OR } \\
\text { peripheral eosinophilia }\end{array}$ & & \\
\hline $\begin{array}{l}\text { Clinical improvement following } \\
\text { daptomycin withdrawal }\end{array}$ & $\begin{array}{l}\text { Clinical improvement following } \\
\text { daptomycin withdrawal }\end{array}$ & & \\
\hline Fever & & & \\
\hline
\end{tabular}

Adapted from references 1 and 2

Abbreviation: BAL bronchoalveolar lavage; $C T$ computed tomography; $C X R$ chest x-ray 
Table 2 Summary of 35 cases of presumed daptomycin-induced eosinophilic pneumonia

\begin{tabular}{|c|c|c|c|c|c|c|c|c|}
\hline Case & $\begin{array}{l}\text { Age/ } \\
\text { Sex }\end{array}$ & Indication & $\begin{array}{l}\text { Dose } \\
\text { (mg/kg/ } \\
\text { day) }\end{array}$ & $\begin{array}{l}\text { DAP } \\
\text { Duration } \\
\text { (wks) }\end{array}$ & Clinical Findings & Objective Findings & Treatment & Outcome \\
\hline \multirow[t]{11}{*}{ Kim [1] (2012) } & $63 / F$ & MSSA spinal osteomyelitis & 6 & 3 & $\begin{array}{l}\text { - Fever } \\
\text { - Cough, } \\
\text { hypoxemia }\end{array}$ & $\begin{array}{l}\text { - BAL }=60-70 \% \\
\text { - Peripheral eosinophilia } \\
\text { - Elevated CPK }\end{array}$ & $\begin{array}{l}\text { - DAP d/c } \\
\text { - Corticosteroids }\end{array}$ & Recovered \\
\hline & $\begin{array}{l}64 / \\
M\end{array}$ & $\begin{array}{l}\text { Osteomyelitis with } \\
\text { bacteremia }\end{array}$ & 5.7 & 4 & $\begin{array}{l}\text { - Fever } \\
\text { - Dyspnea, hypoxia }\end{array}$ & $\begin{array}{l}\text { - } \mathrm{BAL}=44 \% \\
\text { - Peripheral eosinophilia } \\
\text { - Pulmonary infiltrates }\end{array}$ & - DAP d/c & Recovered \\
\hline & $\begin{array}{l}79 / \\
M\end{array}$ & Endocarditis & 6 & 6 & $\begin{array}{l}\text { - Fever, cough, } \\
\text { night sweats } \\
\text { - Dyspnea } \\
\text { requiring MV }\end{array}$ & $\begin{array}{l}\text { - BAL }=9-13 \% \\
\cdot \text { Peripheral eosinophilia } \\
\cdot C T=\text { ground glass opacities } \\
\cdot \text { - Lung biopsy = eosinophilic } \\
\text { pneumonitis }\end{array}$ & $\begin{array}{l}\text { - DAP d/c } \\
\text { - Corticosteroids }\end{array}$ & Improved \\
\hline & $\begin{array}{l}26 / \\
M\end{array}$ & MRSA bacteremia & 7.35 & 1.4 & $\begin{array}{l}\text { - Dyspnea } \\
\text { requiring MV }\end{array}$ & $\begin{array}{l}\text { - BAL not performed } \\
\text { - Peripheral eosinophilia } \\
\text { - Pulmonary infiltrates } \\
\text { - Eosinophils in tracheal aspirate }\end{array}$ & - DAP d/c & Improved \\
\hline & $\begin{array}{l}43 / \\
M\end{array}$ & MRSA osteomyelitis & 6 & $1-2$ & $\begin{array}{l}\text { - Pleuritic pain } \\
\text { - Hypoxia requiring } \\
\mathrm{O}_{2}\end{array}$ & $\begin{array}{l}\text { - BAL not performed } \\
\text { - Peripheral eosinophilia } \\
\text { - } C T=\text { bilateral infiltrates }\end{array}$ & $\begin{array}{l}\text { - DAP d/c } \\
\text { - Given NSAIDs, } \\
\text { meperidine }\end{array}$ & $\begin{array}{l}\text { - Improved } \\
\text { - Residual } \\
\text { infiltrates on } \\
\text { CT s/p } 4 \text { wks }\end{array}$ \\
\hline & $\begin{array}{l}66 / \\
M\end{array}$ & MSSA bacteremia & 6 & 1 & $\begin{array}{l}\text { - Dyspnea } \\
\text { requiring } \mathrm{O}_{2} \\
\text { - Hematemesis }\end{array}$ & $\begin{array}{l}\text { - BAL with eosinophils } \\
\text { (not quantified) } \\
\text { - Peripheral eosinophilia }\end{array}$ & $\begin{array}{l}\text { - DAP d/c } \\
\text { - Corticosteroids }\end{array}$ & Recovered \\
\hline & $\begin{array}{l}71 / \\
M\end{array}$ & $\begin{array}{l}\text { MRSA diabetic foot } \\
\text { infection }\end{array}$ & 4 & 7.7 & $\begin{array}{l}\text { - Dyspnea } \\
\text { requiring } \mathrm{O}_{2}\end{array}$ & $\begin{array}{l}\text { - Peripheral eosinophilia } \\
\text { - Elevated CRP } \\
\text { - Elevated ESR } \\
\cdot \text { - } C \text { = bilateral interstitial opacities }\end{array}$ & - DAP d/c & Improved \\
\hline & $77 / F$ & Bacteremia (enterococcal) & 5 & 1 & $\begin{array}{l}\text { - Dyspnea } \\
\text { requiring } \mathrm{O}_{2}\end{array}$ & $\begin{array}{l}\text { - Peripheral eosinophilia } \\
\text { - CXR = pneumonitis }\end{array}$ & $\begin{array}{l}\text { - DAP d/c } \\
\text { - Corticosteroids }\end{array}$ & Improved \\
\hline & $\begin{array}{l}67 / \\
M\end{array}$ & MRSA endocarditis & 6 & 4.3 & $\begin{array}{l}\text { - Dyspnea } \\
\text { requiring MV }\end{array}$ & $\begin{array}{l}\text { - } \mathrm{BAL}=9 \% \\
\text { - Peripheral eosinophilia } \\
\cdot \mathrm{CT}=\text { bilateral pulmonary } \\
\text { infiltrates }\end{array}$ & $\begin{array}{l}\text { - DAP d/c } \\
\text { - Corticosteroids }\end{array}$ & Improved \\
\hline & $\begin{array}{l}73 / \\
M\end{array}$ & Prosthetic joint infection & 5 & 3.7 & $\begin{array}{l}\text { - Fever } \\
\text { - Dyspnea } \\
\text { requiring MV }\end{array}$ & $\begin{array}{l}\text { - Peripheral eosinophilia } \\
\cdot \mathrm{CT}=\text { bilateral ground glass } \\
\text { appearance }\end{array}$ & $\begin{array}{l}\text { - DAP d/c } \\
\text { - Corticosteroids }\end{array}$ & Recovered \\
\hline & $81 / F$ & MRSA paraspinal abscess & 6 & 1.6 & $\begin{array}{l}\text { - Dyspnea } \\
\text { requiring MV }\end{array}$ & $\begin{array}{l}\text { - BAL }=2 \%(\mathrm{~s} / \mathrm{p} \text { corticosteroid })^{\mathrm{a}} \\
\cdot \mathrm{CXR}=\text { bilateral mid-lung infiltrates }\end{array}$ & $\begin{array}{l}\text { - DAP d/c } \\
\text { - Corticosteroids }\end{array}$ & Improved \\
\hline Cobb [6] (2007) & $\begin{array}{l}84 / \\
M\end{array}$ & $\begin{array}{l}\text { Infection of left knee } \\
\text { prosthesis }\end{array}$ & 4 & 4 & $\begin{array}{l}\text { - Decreased } \\
\text { appetite } \\
\text { - Weight loss } \\
\text { - Fatigue } \\
\text { - Weakness }\end{array}$ & $\begin{array}{l}\text { - Elevated ESR } \\
\cdot \text { CT with infiltrates } \\
\cdot \text { - Lung biopsy = eosinophilic } \\
\text { pneumonia }\end{array}$ & - DAP d/c & $\begin{array}{l}\text { - Improved within } \\
2 \text { weeks }\end{array}$ \\
\hline
\end{tabular}


Table 2 Summary of 35 cases of presumed daptomycin-induced eosinophilic pneumonia (Continued)

\begin{tabular}{|c|c|c|c|c|c|c|c|c|}
\hline Hayes [7] (2007) & $\begin{array}{l}60 / \\
M\end{array}$ & MSSA endocarditis & NR & 2 & $\begin{array}{l}\text { - Fever, rigors, } \\
\text { diaphoresis } \\
\text { - Required MV }\end{array}$ & $\begin{array}{l}\text { - BAL } 16 \% \text { initially } \\
\text { - BAL } 26 \% \text { after rechallenge } \\
\text { - CRP elevated }\end{array}$ & $\begin{array}{l}\text { - DAP d/c - then re- } \\
\text { challenged } \\
\text { - DAP d/c plus } \\
\text { corticosteroids }\end{array}$ & $\begin{array}{l}\text { - Rechallenge failed } \\
\text { within } 4 \mathrm{~h} \\
\text { - Improved within } 24 \mathrm{~h} \\
\text { after DAP d/c }\end{array}$ \\
\hline Kakish [8] (2008) & $\begin{array}{l}65 / \\
M\end{array}$ & $\begin{array}{l}\text { MRSA vertebral osteomyelitis, } \\
\text { epidural abscess }\end{array}$ & 6 & 2 & $\begin{array}{l}\text { - Low-grade fever } \\
\text { - Dyspnea } \\
\text { requiring MV }\end{array}$ & $\begin{array}{l}\text { - BAL }=33 \% \\
\text { - Peripheral eosinophilia } \\
\text { - Lung biopsy revealed eosinophils }\end{array}$ & $\begin{array}{l}\text { - DAP d/c } \\
\text { - Corticosteroids }\end{array}$ & $\begin{array}{l}\text { - Improved within } 72 \mathrm{~h} \\
\text { - Normal CT at } \\
3 \text { months }\end{array}$ \\
\hline Shinde [9] (2009) & $\begin{array}{l}54 / \\
M\end{array}$ & Complicated inguinal hernia repair & NR & 2 & $\begin{array}{l}\text { - Low grade fever, } \\
\text { cough } \\
\text { - Hypoxemia } \\
\text { requiring MV }\end{array}$ & $\begin{array}{l}\text { - Peripheral eosinophilia } \\
\text { - CT = bilateral airspace, peripheral } \\
\text { predominance, small bilateral effusions } \\
\text { - Lung biopsy = many eosinophils }\end{array}$ & $\begin{array}{l}\text { - DAP d/c } \\
\text { - Corticosteroids }\end{array}$ & $\begin{array}{l}\text { - Improved within } 24 \mathrm{~h} \\
\text { - Normal CT at } 4 \text { weeks }\end{array}$ \\
\hline \multirow[t]{2}{*}{ Lal [10] (2010) } & $\begin{array}{l}82 / \\
M\end{array}$ & Prosthetic joint infection & NR & 3 & $\begin{array}{l}\text { - Fever } \\
\text { - Hypoxia requiring } \\
\mathrm{O}_{2}\end{array}$ & $\begin{array}{l}\text { - } \mathrm{BAL}=14 \% \\
\text { - Peripheral eosinophilia } \\
\text { - } \mathrm{CT}=\text { patchy bilateral infiltrates }\end{array}$ & $\begin{array}{l}\text { - DAP d/c } \\
\text { - Corticosteroids }\end{array}$ & $\begin{array}{l}\text { - Recovered after } \\
5 \text { days } \\
\text { - Recurrent symptoms } \\
\text { - Low dose steroids } \\
\text { required }\end{array}$ \\
\hline & $\begin{array}{l}87 / \\
M\end{array}$ & Prosthetic knee infection & NR & 4 & $\begin{array}{l}\text { - Dyspnea, dry } \\
\text { cough requiring } \\
\mathrm{O}_{2} \\
\text { - Malaise, chills, } \\
\text { anorexia, fever }\end{array}$ & $\begin{array}{l}\text { - } \mathrm{BAL}=40 \% \\
\text { - Peripheral eosinophilia } \\
\text { - } \mathrm{CT}=\text { bilateral patchy pulmonary } \\
\text { infiltrates }\end{array}$ & $\begin{array}{l}\text { - DAP d/c } \\
\text { - Corticosteroids }\end{array}$ & $\begin{array}{l}\cdot \text { Recurrence } s / p \\
\text { steroid taper } \\
\cdot \text { Low dose steroids for } \\
2 \text { years }\end{array}$ \\
\hline \multirow[t]{3}{*}{ Miller [11] (2010) } & $\begin{array}{l}60 / \\
M\end{array}$ & MSSA prosthetic hip infection & 6 & 2 & $\begin{array}{l}\text { - Cough, fever } \\
\text { - Hypoxia requiring } \\
\mathrm{O}_{2}\end{array}$ & $\begin{array}{l}\text { - BAL }=81 \% \text { after rechallenge } \\
\text { - Peripheral eosinophilia } \\
\text { - } \mathrm{CT}=\text { bilateral scattered ground-glass } \\
\text { opacities } \\
\text { - Lung biopsy = acute fibrinous and } \\
\text { organizing pneumonia, reactive } \\
\text { alveolar and interstitial epithelial changes }\end{array}$ & $\begin{array}{l}\cdot \text { DAP d/c } \\
\cdot \text { Rechallenged, DAP } \\
\text { d/c } \\
\cdot \text { Corticosteroids }\end{array}$ & $\begin{array}{l}\text { - Improved within } 48 \mathrm{~h} \\
\text { - Rechallenge failed } \\
\text { within } 24 \mathrm{~h}\end{array}$ \\
\hline & $\begin{array}{l}60 / \\
M\end{array}$ & MRSA osteomyelitis, septic arthritis & 6 & 2 & $\begin{array}{l}\text { - Non-productive } \\
\text { cough, dyspnea } \\
\text { - Low-grade fevers, } \\
\text { chills }\end{array}$ & $\begin{array}{l}\text { - Peripheral eosinophilia } \\
\text { - } \mathrm{CT}=\text { patchy peripheral } \\
\text { nodular/ground-glass }\end{array}$ & - DAP d/c & $\begin{array}{l}\text { - Resolution within } \\
96 \mathrm{~h} \\
\text { - Recurrence with re- } \\
\text { challenge at } 5 \text { months }\end{array}$ \\
\hline & $\begin{array}{l}83 / \\
M\end{array}$ & Diskitis of lumbar spine & 6 & 4 & $\begin{array}{l}\text { - Progressive } \\
\text { dyspnea, } \\
\text { - Cough, pleuritic } \\
\text { chest pain }\end{array}$ & $\begin{array}{l}\text { - BAL }=13 \% \\
\text { - Peripheral eosinophilia } \\
\text { - } \mathrm{CT}=\text { diffuse ground-glass, reticular } \\
\text { opacities } \\
\text { - Lung biopsy = acute organizing } \\
\text { pneumonia, eosinophilia, chronic } \\
\text { inflammation, fibro-inflammatory } \\
\text { changes }\end{array}$ & $\begin{array}{l}\text { - DAP d/c } \\
\text { - Corticosteroids }\end{array}$ & $\begin{array}{l}\text { Improved within } \\
6 \text { days }\end{array}$ \\
\hline $\begin{array}{l}\text { Kalogeropoulous } \\
\text { [12] (2011) }\end{array}$ & $\begin{array}{l}78 / \\
M\end{array}$ & Endocarditis & 8 & 1.4 & $\begin{array}{l}\text { - Fever, chills, } \\
\text { diaphoresis, } \\
\text { - Hypoxemia } \\
\text { requiring } \mathrm{O}_{2}\end{array}$ & $\begin{array}{l}\text { - } \text { BAL }=27.5 \% \\
\text { - Peripheral eosinophilia } \\
\text { - Elevated ESR } \\
\text { - Elevated CRP } \\
\text { - CT = patchy consolidation, } \\
\text { ground-glass opacities, bilateral } \\
\text { pleural effusions }\end{array}$ & - DAP d/c & Resolution within $24 \mathrm{~h}$ \\
\hline
\end{tabular}


Table 2 Summary of 35 cases of presumed daptomycin-induced eosinophilic pneumonia (Continued)

\begin{tabular}{|c|c|c|c|c|c|c|c|c|}
\hline $\begin{array}{l}\text { Rether [13] } \\
\text { (2011) }\end{array}$ & $\begin{array}{l}69 / \\
M\end{array}$ & $\begin{array}{l}\text { Spondylo-discitis with lumbar } \\
\text { epidural and bilateral psoas } \\
\text { abscesses }\end{array}$ & 6 & 3 & $\begin{array}{l}\text { - Fever } \\
\text { - Dyspnea } \\
\text { requiring } \mathrm{O}_{2}\end{array}$ & $\begin{array}{l}\text { - BAL }=30 \% \\
\text { - Elevated CRP } \\
\text { - CXR = extensive patchy infiltrates } \\
\text { in RLL and entire left lung }\end{array}$ & $\begin{array}{l}\text { - DAP d/c } \\
\text { - Corticosteroids }\end{array}$ & Improved within $24 \mathrm{~h}$ \\
\hline Patel [14] (2014) & $61 / F$ & Osteomyelitis & NR & 1 & $\begin{array}{l}\text { - Dry cough } \\
\text { - Dyspnea } \\
\text { requiring MV }\end{array}$ & $\begin{array}{l}\text { - } \mathrm{BAL}=30 \% \\
\text { - Peripheral eosinophilia } \\
\text { - } \mathrm{CT}=\text { bilateral pleural effusion, } \\
\text { diffuse bilateral patchy infiltrate }\end{array}$ & $\begin{array}{l}\text { - DAP d/c } \\
\text { - Corticosteroids }\end{array}$ & Improved within $72 \mathrm{~h}$ \\
\hline \multirow[t]{2}{*}{ Phillips [15] 2014) } & $\begin{array}{l}48 / \\
M\end{array}$ & Osteomyelitis & 6 & 3 & $\begin{array}{l}\text { - Fever } \\
\text { - Dyspnea } \\
\text { requiring MV }\end{array}$ & $\begin{array}{l}\text { - BAL }=17 \% \\
\text { - Peripheral eosinophilia } \\
\text { - CXR = patchy bilateral airspace } \\
\text { opacities }\end{array}$ & $\begin{array}{l}\text { - DAP d/c } \\
\text { - Corticosteroids }\end{array}$ & - Improved \\
\hline & $\begin{array}{l}28 / \\
M\end{array}$ & Osteomyelitis & 6 & 4 & $\begin{array}{l}\text { - Dyspnea } \\
\text { requiring MV } \\
\text { - Chest pain, light- } \\
\text { headedness }\end{array}$ & $\begin{array}{l}\text { - BAL }=74 \% \\
\cdot \text { Peripheral eosinophilia } \\
\cdot C T=\text { diffuse multi-lobar infiltrates }\end{array}$ & $\begin{array}{l}\text { - DAP d/c } \\
\text { - Corticosteroids }\end{array}$ & $\begin{array}{l}\text { Resolution within } \\
1 \text { week }\end{array}$ \\
\hline $\begin{array}{l}\text { Yamamoto [16] } \\
\text { (2014) }\end{array}$ & $\begin{array}{l}82 / \\
M\end{array}$ & MRSA bacteremia & 10 & 2 & $\begin{array}{l}\cdot \text { Low grade fever } \\
\cdot \text { Hypoxia }\end{array}$ & $\begin{array}{l}\cdot \mathrm{CT}=\text { bilateral ground glass opacities } \\
\text { - Sputum negative for eosinophils }\end{array}$ & - DAP d/c & Improved \\
\hline \multirow[t]{2}{*}{ Yusuf [17] (2014) } & $\begin{array}{l}64 / \\
M\end{array}$ & Prosthetic joint infection & 10 & 4 & - Fever & $\begin{array}{l}\text { - BAL }=47 \% \\
\text { - Peripheral eosinophilia } \\
\text { - Elevated CRP } \\
\text { - } \mathrm{CT}=\text { diffuse bilateral ground-glass } \\
\text { opacities }\end{array}$ & - DAP d/c & Improved within $24 \mathrm{~h}$ \\
\hline & $\begin{array}{l}61 / \\
M\end{array}$ & Prosthetic joint infection & 10 & 2 & $\begin{array}{l}\text { - Fever } \\
\text { - Dyspnea } \\
\text { requiring MV }\end{array}$ & $\begin{array}{l}\text { - } \mathrm{BAL}=3 \% \\
\text { - Peripheral eosinophilia } \\
\text { - Elevated CRP } \\
\cdot \mathrm{CT}=\text { ground-glass consolidation, } \\
\text { bilateral pleural effusion }\end{array}$ & - DAP d/c & Improved within $24 \mathrm{~h}$ \\
\hline \multirow[t]{2}{*}{ Chiu [18] (2015) } & $\begin{array}{l}77 / \\
M\end{array}$ & Osteomyelitis & 6 & 6 & $\begin{array}{l}\text { - Pleuritic chest } \\
\text { pain } \\
\cdot \text { Cough, dyspnea } \\
\text { requiring } \mathrm{O}_{2}\end{array}$ & $\begin{array}{l}\text { - } \mathrm{BAL}=18 \% \\
\text { - Elevated CRP } \\
\cdot \text { CXR }=\text { diffuse bilateral airspace disease }\end{array}$ & $\begin{array}{l}\text { - DAP was } \mathrm{d} / \mathrm{c} 1 \text { day } \\
\text { before symptoms } \\
\text { - Corticosteroids }\end{array}$ & Improved within $60 \mathrm{~h}$ \\
\hline & $74 / F$ & Infected hip reconstruction & 6 & $1^{b}$ & $\begin{array}{l}\text { - } \text { Fever } \\
\text { - Dyspnea } \\
\text { requiring } \mathrm{O}_{2}\end{array}$ & - CXR - bilateral airspace disease & $\begin{array}{l}\text { - DAP d/c } \\
\text { - Corticosteroids }\end{array}$ & Improved within $24 \mathrm{~h}$ \\
\hline $\begin{array}{l}\text { Hagiya [19] } \\
(2015)\end{array}$ & $\begin{array}{l}34 / \\
M\end{array}$ & Endocarditis & 10 & 1 & $\begin{array}{l}\text { - Cough with mild } \\
\text { hypoxemia }\end{array}$ & $\begin{array}{l}\text { - Peripheral eosinophilia } \\
\text { - Elevated CRP } \\
\cdot \mathrm{CT}=\text { consolidation in peripheral } \\
\text { field of right upper lobe }\end{array}$ & - DAP d/c & $\begin{array}{l}\text { Resolved within } \\
6 \text { weeks }\end{array}$ \\
\hline $\begin{array}{l}\text { Hatipoglu [20] } \\
\text { (2015) }\end{array}$ & $67 / F$ & MRSA diabetic foot ulcer & $N R^{c}$ & 3.3 & $\begin{array}{l}\text { - Cough, dyspnea } \\
\text { requiring BPAP } \\
\text { - Fever, fatigue, } \\
\text { decreased appetite }\end{array}$ & $\begin{array}{l}\text { - Peripheral eosinophilia } \\
\text { - Elevated CRP } \\
\text { - } C T=\text { right lobe infiltration }\end{array}$ & $\begin{array}{l}- \text { DAP d/c } \\
\text { - Inhaled } \\
\text { corticosteroids }\end{array}$ & Improved within $72 \mathrm{~h}$ \\
\hline Roux [21] (2015) & $\begin{array}{l}67 / \\
M\end{array}$ & $\begin{array}{l}\text { MSSA prosthetic hip } \\
\text { infection }\end{array}$ & 6 & 2.4 & $\begin{array}{l}\text { - Dry cough, } \\
\text { hypoxemia }\end{array}$ & $\begin{array}{l}\text { - BAL }=10 \% \\
\text { - Peripheral eosinophilia }\end{array}$ & $\begin{array}{l}\text { - DAP d/c } \\
\text { - Corticosteroids }\end{array}$ & Improved within $96 \mathrm{~h}$ \\
\hline
\end{tabular}


Table 2 Summary of 35 cases of presumed daptomycin-induced eosinophilic pneumonia (Continued)

\begin{tabular}{|c|c|c|c|c|c|c|c|c|}
\hline & & & & & & $\begin{array}{l}\mathrm{CT}=\text { diffuse alveolar and } \\
\text { interstitial opacities }\end{array}$ & & \\
\hline $\begin{array}{l}\text { Wojtaszczyk [22] } \\
\text { (2015) }\end{array}$ & $\begin{array}{l}76 / \\
M\end{array}$ & $\begin{array}{l}\text { Septic arthritis and } \\
\text { pacemaker vegetation }\end{array}$ & $N R$ & 2 & $\begin{array}{l}\text { - Dyspnea } \\
\text { requiring } \mathrm{O}_{2} \\
\text { cough } \\
\text { - Fever, fatigue }\end{array}$ & $\begin{array}{l}\text { - } \mathrm{BAL}=58 \% \\
\text { - Elevated CRP } \\
\text { - } \mathrm{CT}=\text { bilateral ground glass } \\
\text { opacity, patchy consolidation }\end{array}$ & $\begin{array}{l}\text { - DAP d/c } \\
\text { - Corticosteroids }\end{array}$ & Resolved within $72 \mathrm{~h}$ \\
\hline $\begin{array}{l}\text { Akcaer [23] } \\
(2016)\end{array}$ & $\begin{array}{l}60 / \\
M\end{array}$ & $\begin{array}{l}\text { MSSA post-amputation } \\
\text { abscess }\end{array}$ & 5 & 3.4 & $\begin{array}{l}\text { - Tachypnea, } \\
\text { hypoxia requiring } \\
\mathrm{O}_{2}\end{array}$ & $\begin{array}{l}\text { - Peripheral eosinophilia } \\
\text { - Elevated CRP } \\
\text { - Elevated ESR } \\
\text { - HRCT = right pleural effusion, } \\
\text { bilateral tree-in-bud pattern, } \\
\text { bilateral scattered ground-glass } \\
\text { opacities }\end{array}$ & - DAP d/c & Resolved within $72 \mathrm{~h}$ \\
\hline
\end{tabular}

KEY: BAL bronchoalveolar lavage, BPAP bilevel positive airway, CPK creatine phosphokinase, CRP C-reactive protein, CT computed tomography scan, CXR chest x-ray, DAP daptomycin, $d / C$ discontinued, ESR erythrocyte sedimentation rate, $F$ female, HRCT high resolution computed tomography, $M$ male, MRSA methicillin resistant Staphylococcus aureus, MSSA methicillin susceptible Staphylococcus aureus, MV mechanical ventilation, NR not reported, NSAID nonsteroidal anti-inflammatory drug, $\mathrm{O}_{2}$ oxygen, $R L L$ right lower lobe; $s / p$ status post, wks weeks

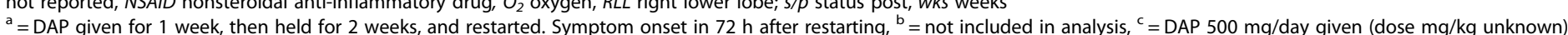


Analysis of the 35 cases shows eosinophilic pneumonia resulting from daptomycin use is most likely to be reported in males with a mean age of $65.4 \pm 15$ years and a mean length of therapy of $2.8 \pm 1.6$ weeks at symptom onset. The most common indication for daptomycin use was osteomyelitis and/or diabetic foot infection closely followed by prosthetic joint infection. Daptomycin dose ranged from 4 to $10 \mathrm{mg} / \mathrm{kg} /$ day depending on renal function; therefore, the adverse effect does not appear to be dose dependent, but time dependent exposure. The most common symptoms of eosinophilic pneumonia included fever and dyspnea often requiring either oxygen supplementation or mechanical ventilation. Other clinical findings included malaise, elevated ESR (4/35 cases), or elevated C-reactive protein (11/35 cases). Peripheral eosinophilia was also present in approximately $77 \%$ (27/35 cases) of patients. Many cases also had computed tomography scans or chest $\mathrm{x}$-rays which revealed opacities (12/35 cases) and bilateral infiltrates (13/35 cases). Symptom improvement was seen within $24 \mathrm{~h}$ through one week after daptomycin discontinuation. The majority of patients were also prescribed treatment with corticosteroids (23/35 cases); however, all patients were reported to recover (See Table 3).

\section{Discussion}

While the criteria developed by Solomon and Schwartz differ from the FDA guidance, they are largely similar in that an offending agent (here, daptomycin) must be present, $>25 \%$ eosinophils are present, and that clinical improvement is seen after discontinuation of the drug. The FDA guidance also includes some measures of symptomatology such as fever and dyspnea [2, 4].

Overall, dyspnea was the most common documented symptom associated with eosinophilic pneumonia followed by the presence of either pulmonary infiltrates or opacities on chest x-ray or CT. A total of 10 cases specifically mentioned the characteristic finding of ground glass opacities on CT. A potential limitation of this review is that some of the daptomycin-induced eosinophilic pneumonia used lung biopsy in place of BAL as part of the diagnostic criteria which is not part of the FDA guidance, but is included in the Solomon and Schwartz criteria. In addition, since some patients had $\mathrm{BAL}<25 \%$ eosinophils but lung biopsy revealed eosinophilia $[1,13]$, a $25 \%$ cut off may be too strict in certain situations.

Corticosteroids are believed to be beneficial at halting clinical manifestations of daptomycin-induced eosinophilic pneumonia and were used in the majority of reported cases. Steroids exert action through eosinophilic apoptosis and through accelerating intracellular signaling involved in eosinophil death [45]. No dose or length of
Table 3 Compilation of available data on 35 cases of daptomycin-induced eosinophilic pneumonia

\begin{tabular}{|c|c|}
\hline \multicolumn{2}{|l|}{ Sex, n (\%) } \\
\hline Male & $29(83)$ \\
\hline Female & $6(17)$ \\
\hline Age(years), mean $\pm S D$ & $65.4 \pm 15$ \\
\hline \multicolumn{2}{|l|}{ Daptomycin indication, n (\%) } \\
\hline Osteomyelitis/diabetic foot infection & $11(31)$ \\
\hline Prosthetic joint infection & $9(26)$ \\
\hline Endocarditis & $5(14)$ \\
\hline Bacteremia & $4(11)$ \\
\hline Abscess & $3(9)$ \\
\hline Other & $3(9)$ \\
\hline $\begin{array}{l}\text { Daptomycin dose (mg/kg/day), } \\
\text { mean } \pm S D\end{array}$ & $6.4 \pm 1.6$ \\
\hline $\begin{array}{l}\text { Treatment duration at symptom } \\
\text { onset (weeks), mean } \pm \text { SD }\end{array}$ & $2.8 \pm 1.6$ \\
\hline \multicolumn{2}{|l|}{ Clinical findings, $\mathrm{n}(\%)$} \\
\hline Dyspnea & $33(94)$ \\
\hline Fever & $20(57)$ \\
\hline Cough & $13(37)$ \\
\hline Requiring oxygen & $15(43)$ \\
\hline Requiring mechanical ventilation & $12(34)$ \\
\hline Infiltrates/opacities of CT/CXR, n (\%) & $30(86)$ \\
\hline BAL eosinophils $\%$, mean \pm SD & $32 \pm 22.4$ \\
\hline Peripheral eosinophilia, n (\%) & $27(77)$ \\
\hline Lung biopsy consistent with AEP, n (\%) & $6(17)$ \\
\hline \multicolumn{2}{|l|}{ Treatment, n (\%) } \\
\hline Daptomycin discontinued only & $12(34)$ \\
\hline Daptomycin discontinued plus corticosteroid & $23(66)$ \\
\hline
\end{tabular}

$A E P$ acute eosinophilic pneumonia, $B A L$ bronchoalveolar lavage, $C T$ computed tomography, CXR chest $\mathrm{x}$-ray, SD standard deviation

corticosteroid treatment is established in guidelines for eosinophilic pneumonia; however, a commonly employed regimen is intravenous methylprednisolone 60-125 mg every $6 \mathrm{~h}$, with conversion to prednisone 40-60 $\mathrm{mg}$ oral daily and taper over 2-6 weeks. Use of a 2- or 4-week course appears to have similar time to resolution of clinical symptoms and radiological abnormalities [46].

\section{Conclusion}

As use of daptomycin continues to increase, it is important for clinicians to recognize and appropriately manage daptomycin-induced eosinophilic pneumonia. Although symptoms may resolve upon discontinuation of daptomycin, use of corticosteroid may be beneficial for recovery. Further research is needed to determine the exact mechanism of daptomycin-induced eosinophilic pneumonia and identify the optimal treatment course. 


\section{Abbreviations}

AERS: Aderverse Event Reporting System; BAL: Bronchoalveolar lavage; CT: Computed tomography; CXR: Chest X-ray; EP: Eosinophilic pneumonia; ESR: Erythrocyte sedimentation rate; FDA: Food and Drug Administration; MRSA: Methicillin resistant Staphylococcus aureus; VRE: Vancomycin resistant enterococcus

\section{Acknowledgements}

Not applicable.

\section{Funding}

The views expressed are those of the authors and do not necessarily reflect the position or policy of the United States Department of Veterans Affairs. This material is based upon work supported, in part, by the Office of Research and Development, Department of Veterans Affairs.

\section{Availability of data and materials}

Data sharing not applicable to this article as no datasets were generated or analyzed during the current study.

\section{Authors' contributions}

PU initiated the project, participated in review of literature, and was a major contributor in writing of the manuscript. KL provided analysis of intellectual content, writing of the manuscript, and critical revision. MG and MJ provided analysis of intellectual content and writing the manuscript. KW participated in review of literature, writing of the manuscript, and critical revision. All authors read and approved the final manuscript.

\section{Competing interests}

The views expressed are those of the authors and do not necessarily reflect the position or policy of the United States Department of Veterans Affairs. Kerry L. LaPlante has received research funding or acted as an advisor, or consultant for Cubist, Davol, Forest, and Pfizer Inc. Kristina Ward has received research funding from Pfizer Inc. Melissa Gaitanis, Matthew Jankowich, Priyasha Uppal report no conflicts.

\section{Consent for publication}

Not applicable

\section{Ethics approval and consent to participate}

Not applicable_-systematic review of published literature.

\section{Author details}

${ }^{1}$ Providence Veterans Affairs Medical Center, Providence, RI, USA 2Department of Pharmacy Practice, University of Rhode Island, 7 Greenhouse Rd, Suite 295 J, Kingston, RI 02881, USA. ${ }^{3}$ Warren Alpert Medical School of Brown University, Providence, RI, USA.

Received: 12 August 2016 Accepted: 29 November 2016

Published online: 12 December 2016

\section{References}

1. Kim PW, Sorbello AF, Wassel RT, et al. Eosinophilic pneumonia in patients treated with daptomycin: review of the literature and US FDA adverse event reporting system records. Drug Saf. 2012:35:447-57.

2. US Food and Drug Administration. FDA drug safety communication: eosinophilic pneumonia associated with the use of Cubicin (daptomycin). http://www.fda.gov/Drugs/DrugSafety/

PostmarketDrugSafetylnformationforPatientsandProviders/ucm220273.htm. (Accessed 30 Nov 2016).

3. Allen JN. Drug-induced eosinophilic lung disease. Clin Chest Med. 2004;25:77-88.

4. Cubicin (daptomycin) package insert. Lexington, MA: Cubist Pharmaceuticals; 2006 May.

5. Silverman JA, Mortin LI, Vanpraagh AD, et al. Inhibition of daptomycin by pulmonary surfactant: in vitro modeling and clinical impact. J Infect Dis. 2005:191:2149-52.

6. Cobb E, Kimbrough RC, Nugent KM, Phy MP. Organizing pneumonia and pulmonary eosinophilic infiltration associated with daptomycin. Ann Pharmacother. 2007;41:696-701.

7. Hayes Jr D, Anstead MI, Kuhn RJ. Eosinophilic pneumonia induced by daptomycin. J Infect. 2007;54:e211-3.
8. Kakish E, Wiesner AM, Winstead PS, Bensadoun ES. Acute respiratory failure due to daptomycin induced eosinophilic pneumonia. Respir Med. 2008;1:235-7.

9. Shinde A, Seifi A, DelRe S, et al. Daptomycin-induced pulmonary infiltrates with eosinophiiia. J Infect. 2009;58:173-4.

10. Lal Y, Assimacopoulos AP. Two cases of daptomycin induced eosinophilic pneumonia and chronic pneumonitis. Clin Infect Dis. 2010;50:737-40.

11. Miller BA, Gray A, Leblanc TW, Sexton DJ, et al. Acute eosinophilic pneumonia secondary to daptomycin: a report of three cases. Clin Infect Dis. 2010;50:e63-8.

12. Kalogeropoulos AS, Tsiodras S, Loverdos D, et al. Eosinophilic pneumonia associated with daptomycin: a case report and a review of the literature. Med Case Rep. 2011:5:13. doi:10.1186/1752-1947-5-13.

13. Rether C, Conen A, Grossenbacher M, Albrich WC. A rare cause of pulmonary infiltrates one should be aware of: a case of daptomycininduced acute eosinophilic pneumonia. Infection. 2011;39:583-5.

14. Patel JJ, Atony A, Herrera M, Lipchik RJ. Daptomycin-induced acute eosinophilic pneumonia. WMJ. 2014;113:199-201.

15. Phillips J, Cardile AP, Oatterson TF, Lewis II JS. Daptomycin-induced acute eosinophilic pneumonia: Analysis of the current data and illustrative case reports. Scand J Infect Dis. 2013:45:804-8.

16. Yamamoto K, Hayakawa K, Ohmagari N. Daptomycin-induced pneumonitis in a patient with chronic obstructive pulmonary disease (COPD). Intern Med. 2014;53:2559-60. doi:10.2169/internalmedicine.53.3058.

17. Yusuf E, Perrottet N, Orasch C, et al. Daptomycin-associated eosinophilic pneumonia in Two patients with prosthetic joint infection. Surg Infect. 2014 15:834-7.

18. Chiu SY, Faust AC, Dand HM. Daptomycin-induced eosinophilic pneumonia treated with intravenous corticosteroids. J Pharm Pract. 2015;28:275-9.

19. Hagiya H, Hasegawa K, Asano K, et al. Myopathy and eosinophilic pneumonia coincidentally induced by treatment with daptomycin. Intern Med. 2015:54:525-9. doi:10.2169/internalmedicine.54.3397.

20. Hatipoglu M, Memis A, Turhan V et al. Possible daptomycin-induced acute eosinophilic pneumonia in a patient with diabetic foot infection. Int J Antimicrob Agents. 2016;47:414-5. doi: 10.1016/j.jjantimicag.2016.02.014

21. Roux S, Ferry T, Chidiac C, Valour F. Daptomycin-induced eosinophilic pneumonia. Int J Infect Dis. 2015;37:95-6.

22. Wojtaszczyk A, Jankowich M. Dyspnea on daptomycin: eosinophilic pneumonia. RI Med J. 2015;98:41-3.

23. Akcaer M, Karakas A, Tok D et al. Eosinophilic pneumonia: daptomycininduced lung complication. Med Mal Infect. 2016 http://dx.doi.org/10.1016/j. medmal.2016.01.006

24. Prahl JD, Tripp MS, Stafford CM. Organizing pneumonia and pneumothorax associated with daptomycin use. Chest. 2010; 138 (4) Suppl: 77A1-2. American College of Chest Physicians Annual Meeting October 30 - November 4, 2010; Vancouver, British Columbia, Canada. doi: 10.1378/chest.9330

25. Brixey AG, Willers ED, Ferrel P, Light RW. Daptomycin-induced eosinophilic pneumonia. Am J Resp Crit Care Med. 2011; 183(1): A3876. American Thoracic Society 2011 International Conference, May 13-18, 2011; Denver, CO doi: 10.1164/ajrccm-conference.2011.183.1_MeetingAbstracts.A3876

26. Chow L, Gorga J, Zein J. Development of new pulmonary infiltrates in a patient treated with daptomyinc. Chest. 2011:140(4 Meeting Abstracts): 159A American College of Chest Physicians Annual Meeting October 22-26, 2011; Honolulu, Hawaii. doi:10.1378/chest.1117499.

27. Gaines J, Mills A. Daptomycin-induced eosinophilic pneumonia. J Hosp Med. 2011:6 (4 Suppl 2): S181-182. Society of Hospital Medicine 2011 Abstracts Research, Innovations, Clinical Vignettes Competition, Hospital Medicine, May 10-13, 2011; Grapevine, Texas. doi: 10.1002/jhm.920

28. O'Brien C. Acute eosinophilic pneumonia secondary to daptomycin: A potentially fatal adverse drug reaction. J Hosp Med. 2011:6 (4 Suppl 2): S224-225. Society of Hospital Medicine 2011 Abstracts Research, Innovations, Clinical Vignettes Competition, Hospital Medicine, May 10-13, 2011; Grapevine, Texas. doi: 10.1002/jhm.920

29. Mehta P, Wong W, Ramalingam S et al. Chest. 2012:142(4_Meeting Abstracts):1042A. American College of Chest Physicians Annual Meeting October 20-25, 2012; Atlanta, Georgia. doi: 10.1378/chest.1380652.

30. Safadi B, Carlos W, Bosslet G. Daptomycin-induced eosinophilic pneumonia. Chest. 2012:142(4_Meeting Abstracts):485A. American College of Chest Physicians Annual Meeting October 20-25, 2012; Atlanta, Georgia. doi:10. 1378/chest.1388121. 
31. Tolle LB, Hyzy RC. A 73-year-old man with acute eosinophilic pneumonia owing to daptomycin. Am J Resp Crit Care Med. 2012;185:A4620. American Thoracic Society 2012 International Conference, May 18-23, 2012; San Francisco, California. doi: 10.1164/ajrccm-conference.2012.185.1_ MeetingAbstracts.A4620

32. Edukulla J, Choudhary S, Glick A, DeSouza D. Eosinophilic pneumonia, rare but fatal side effect of daptomycin: A case report. J Gen Intern Med. 2013;28 (Suppl 1):S324. 36th Annual Meeting of the Society of General Internal Medicine April 24-27, 2013; Denver, Colorado. doi: 10. 1007/s1 1606-013-2436-y.

33. Azam M, Asghar S, Aggen D et al. Acute eosinophilic pneumonia: A rare side effect of daptomycin. Chest. 2014; 146 (4_Meeting Abstracts):179A. American College of Chest Physicians Annual Meeting October 25-30, 2014; Austin, Texas. doi:10.1378/chest.1989619.

34. Folkard C, Munoz R. Daptomycin-induced eosinophilic pneumonia. J Gen Intern Med. 2014;29 (Suppl 1): S341-342. 37th Annual Meeting of the Society of General Internal Medicine, April 23-26, 2014; San Diego, California. doi: 10.1007/s11606-014-2834-9.

35. Neyra K, Rahman A, Gupta, V, Cohen Z, Nafees. Acute eosinophilic pneumonia due to exposure to daptomycin leading to ventilatordependent respiratory failure. Chest. 2014;148 (4_Meeting Abstracts):635A. American College of Chest Physicians Annual Meeting October 25-28, 2015: Montreal, Quebec, Canada. doi: 10.1378/chest.2247139

36. Rajagopal A, Mintz E, Reese L. Daptomycin-induced eosinophilic pneumonia without Peripheral eosinophilia. Chest. 2014;145(3_MeetingAbstracts):127A. CHEST World Congress 2014, March 21-24, 2014; Madrid, Spain. doi: 10. 1378/chest.1775453

37. Kuchlean D, le S, Foroozesh M. Daptomycin a pulmonary foe. Am J Respir Crit Care Med. 2015;191:A1661. American Thoracic Society 2015 International Conference, May 15-20, 2015; Denver, Colorado. doi: 10.1164/ ajrccm-conference.2015.191.1_MeetingAbstracts.A1661

38. Hilal T, Khosravi M. Daptomycin-induced acute eosinophilic pneumonia. Am J Respir Crit Care Med. 2015;191:A1533. American Thoracic Society 2015 International Conference, May 15-20, 2015; Denver, Colorado. doi: 10.1164/ ajrccm-conference.2015.191.1_MeetingAbstracts.A1533

39. Goyal P, Breen MJ, Hountras $P$ et al. Daptomycin-induced acute eosinophilic pneumonia: Case report and review of literature. Am J Respir Crit Care Med. 2016; 193:A1658. American Thoracic Society 2016 International Conference, May 13-18, 2016; San Francisco, California. doi: 10.1164/ajrccm conference. 2016.193.1_MeetingAbstracts.A1658.

40. Hirai J, Kinjo T, Hagihara M et al. Eosinophilic pneumonia caused by daptomycin: Five case reports and review of the literature. Am J Respir Crit Care Med. 2016;193:A1586. American Thoracic Society 2016 International Conference, May 13-18, 2016; San Francisco, California. doi: 10.1164/ajrccmconference.2016.193.1_MeetingAbstracts.A1586

41. Komur S, Ulu A, Kurtaran B et al. [Sudden respiratory failure and eosinophilic pneumonia in patients treated with daptomycin: a report of five cases]. Cukurova Med J. 2016; 41): 396-399. doi:10.17826/cutf.208261. Turkish

42. Montenegro O, Del Campo R, Del Rio JJ, Ambrós Checa A. [Acute eosinophilic pneumonia secondary to daptomycin]. Enferm Infecc Microbiol Clin. 2015 Oct 31. doi: 10.1016/j.eimc.2015.08.010. Spanish.

43. Søndergaard TS, Schumacher H, Norup K. Possible daptomycin-induced organizing pneumonia. Ugeskr Laeger. 2010;172:2172-4. Danish.

44. Solomon J, Schwarz M. Drug-, toxin-, and radiation therapy induced eosinophilic pneumonia. Semin Respir Crit Care Med. 2006;27:192-7.

45. Druilhe A, Létuvé S, Pretolani M. Glucocorticoid-induced apoptosis in human eosinophils: mechanism of action. Apoptosis. 2003:8:481-95.

46. Rhee CK, Min KH, Yim NY, et al. Clinical characteristics and corticosteroid treatment of acute eosinophilic pneumonia. Eur Respir J. 2013:41:402.

\section{Submit your next manuscript to BioMed Central and we will help you at every step:}

- We accept pre-submission inquiries

- Our selector tool helps you to find the most relevant journal

- We provide round the clock customer support

- Convenient online submission

- Thorough peer review

- Inclusion in PubMed and all major indexing services

- Maximum visibility for your research

Submit your manuscript at www.biomedcentral.com/submit
Biomed Central 